\title{
Lessons Learned from an Extreme Heat Event using ACES for Situational Awareness, Ontario, Canada
}

\author{
Nancy VanStone, Adam van Dijk, Paul Belanger, Kieran Moore
}

KFL\&A Public Health, Canada

Objective

To describe the lessons learned for public health decision-makers from an analysis of Acute Care Enhanced Surveillance (ACES) data for the heatwaves experienced in Ontario, Canada in the summer of 2018.

\section{Introduction}

The Acute Care Enhanced Surveillance (ACES) system provides syndromic surveillance for Ontario's acute care hospitals. ACES receives over $99 \%$ of acute care records for emergency department (ED) visits; mean daily volume is 17,500 visits. ACES uses a maximum entropy classifier and generates more than 80 standard syndromes, fifteen of which are actively monitored for aberrational activity and are considered of higher public health relevance, including RESP (respiratory infection, non-croup), ILI (influenza-like illness), TOX (toxicological, chemical/drug exposure), AST (asthma), OPI (opioid exposure), CELL (cellulitis), GASTRO (gastroenteritis), ENVIRO (environmental, heat/cold exposure), MH (mental health), EOH (alcohol intoxication), DERM (rash), and SEP (bacteremia, sepsis).

Syndromic surveillance provides a salient source of public health surveillance during extreme heat events; monitoring real-time ED visits can inform local public health authorities of health impacts, provide situation awareness to initiate and/or inform public health response, and help decision-makers allocate resources according to geographic (or demographic) vulnerability. While the use of syndromic surveillance has been well-characterized to monitor infectious disease outbreaks, its use to monitor the heathealth impacts is relatively novel for ACES users, specifically local public health authorities. This report describes the data collected during an extended extreme heat event in Ontario, Canada, to highlight the value of syndromic surveillance during extreme heat events and make recommendations regarding incorporating ACES data into routine workflows.

\section{Methods}

Temperature data were retrieved from Environment Canada historical databases for mid-June to mid-July 2018. Aggregate counts per day for total ED visits and and for individual syndromes were retrieved from ACES databases. Descriptive statistics were used to analyze all datasets.

\section{Results}

An extreme heat event occurred in the southern region of Ontario in early summer, 2018. Environment Canada issues heat warnings for regions throughout Canada according to region-specific criteria; for southern Ontario, heat warnings are issued when 2 or more consecutive days of daytime maximum temperatures are expected to reach $31^{\circ} \mathrm{C}$ or when 2 or more consecutive days of humidex values are expected to reach 40. Extended heat warnings are issued when the event lasts beyond 2 days. An extended heat event occurred June 29 to July 5, 2018. Although the region is large, temperature data from Environment Canada's climate monitoring station at Toronto's Pearson Airport are shown (Figure 1) as an example of the temperatures observed for this time period in the region.

\section{Conclusions}

Lessons learned from an analysis of ACES data during an extreme heat event:

1. The ENVIRO syndrome provides real-time monitoring of the health impacts during a heat event and may provide proxy for estimating the indirect effects of heat (e.g., impacts on chronic conditions). Public health authorities can monitor local health impacts during an extreme heat event.

2. Patients seeking help at the ED do not appear to be skewed in acuity, sex nor age. This does not necessarily reflect the population that experiences the greatest impact from extreme heat, but rather those that are seeking help at the ED for the direct effects of heat. That said, an increase in ENVIRO counts does not indicate whether the

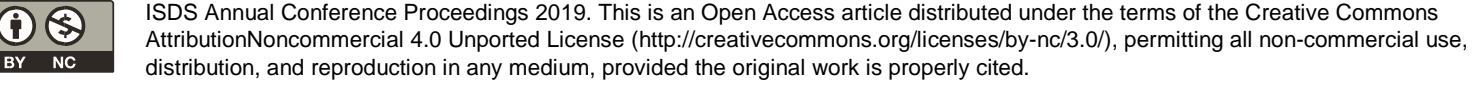


ISDS 2019 Conference Abstracts

increase is due to greater exposure to the heat (or sun), engaging in vigorous outdoor activity during the event (recreational or occupational), or lack of access to air conditioning.

3. ED visits for ENVIRO can be geolocated to determine areas experiencing greater health impacts. This may allow allocation of resources to specifically address vulnerabilities. ACES has built-in mapping capabilities that allows a geovisualization of the home addresses for patients. Furthermore, aggregate counts for relevant syndromes are available for registered users on the Public Health Information Management System (PHIMS), a web-accessible GIS tool for situational awareness that gives public health decision-makers access to real time health impacts in concert with demographics, weather, and other emergency management information.

\section{Acknowledgements}

ACES receives ongoing support from the Government of Ontario's Ministry of Health and Long-Term Care.

\section{Figure 1}

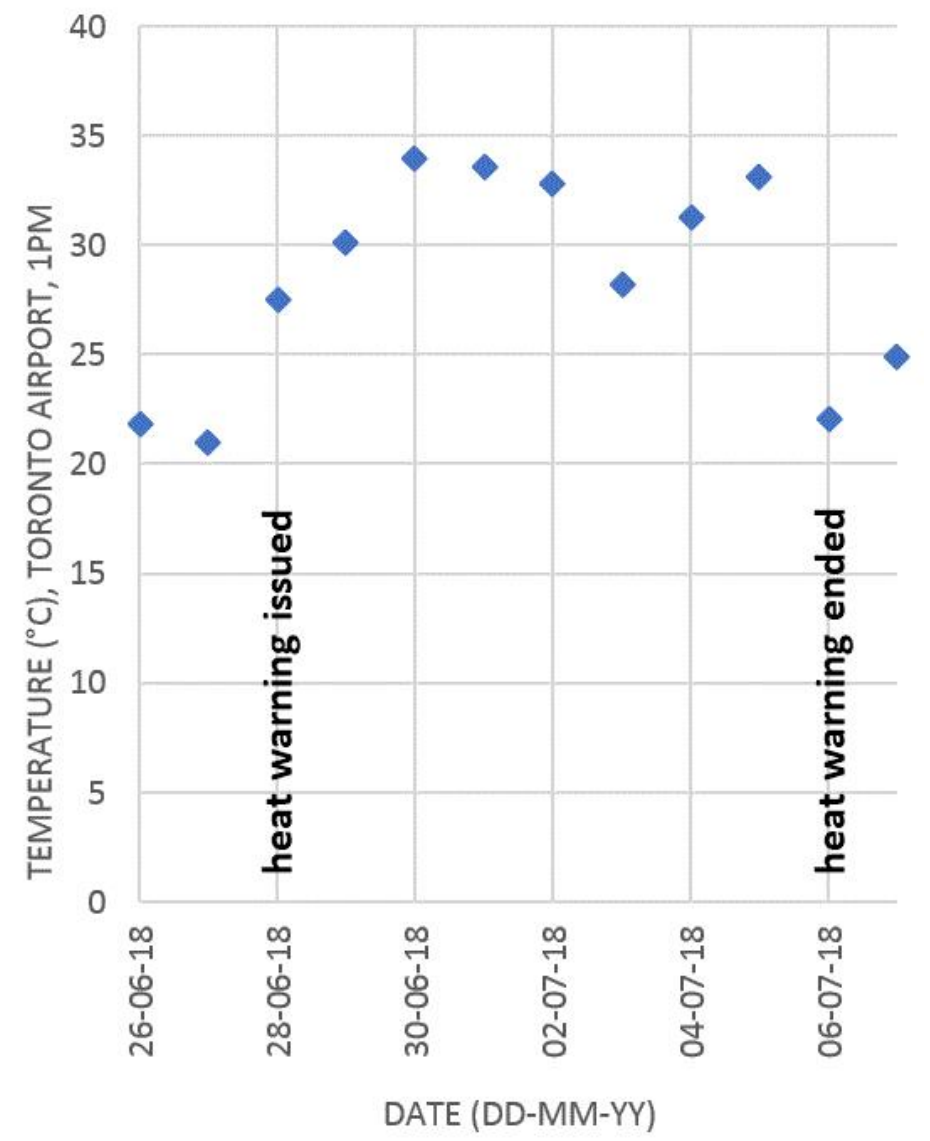

SDS Annual Conference Proceedings 2019. This is an Open Access article distributed under the terms of the Creative Commons AttributionNoncommercial 4.0 Unported License (http://creativecommons.org/licenses/by-nc/3.0/), permitting all non-commercial use, distribution, and reproduction in any medium, provided the original work is properly cited. 


\begin{tabular}{lcccc}
\hline Syndrome & Actual Counts & Expected Counts & Excess Counts & Excess Counts (\%) \\
\hline ALL & 161,561 & 157,917 & 3,644 & 2.3 \\
ENVIRO & 200 & 45 & 155 & 344.4 \\
RESP & 9,677 & 8,316 & 1,361 & 16.4 \\
ILI & 4,406 & 3,780 & 626 & 16.6 \\
DERM & 5,218 & 4,572 & 646 & 14.1 \\
LAC & 5,492 & 4,761 & 731 & 15.4 \\
BITE & 1,890 & 1,467 & 423 & 28.8 \\
\hline
\end{tabular}

60

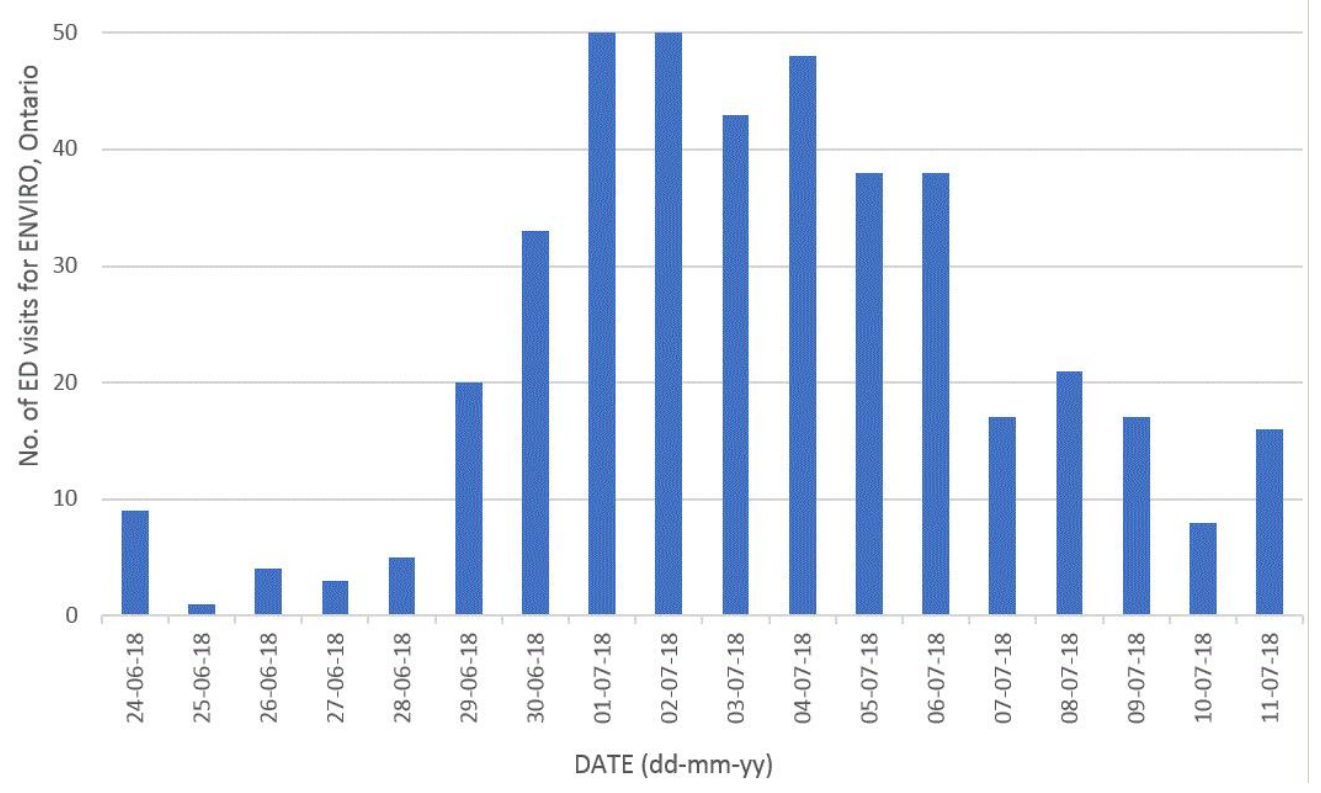

